\title{
Intestinal phenotypic expression of gastric depressed adenomas and the surrounding mucosa
}

\author{
Masafumi Oya ${ }^{1}$, Takashi YaO $^{2}$, Toshihiko Nakamura ${ }^{2}$, Kazuo Nakanishi ${ }^{1}$, and Masazumi Tsuneyoshi ${ }^{2}$ \\ ${ }^{1}$ Department of Pathology, Aso-Iizuka Hospital, Iizuka, Fukuoka, Japan \\ ${ }^{2}$ Department of Anatomic Pathology, Graduate School of Medical Sciences, Kyushu University, 3-1-1 Maidashi, Higashi-ku, Fukuoka \\ 812-8582, Japan
}

\begin{abstract}
Background. Gastric depressed adenomas (DAs) have been reported to be more aggressive than nondepressed ones. To clarify any differences among them, we investigated the intestinal phenotypic expression of DAs and the surrounding mucosa.

Methods. Fifty DAs resected surgically or endoscopically were studied. Forty-two nondepressed adenomas (Non-DAs) were selected as controls. According to the classification of intestinal metaplasia, the phenotypes of the adenomas and surrounding mucosa were classified into four categories: complete intestinal type (I/Com-type), incomplete intestinal type (I/In-type), gastric type (G-type), and unclassified type (U-type), based on a combination of the expression of the antibodies CD10 (small-intestinal brush border), MUC2 (intestinal goblet cells) and HGM (gastric foveolar epithelium). Results. I/In-type adenomas were frequently observed among DAs $(60 \%)$, whereas I/Com-type adenomas were frequently seen in Non-DAs $(52 \%)$. The incidence of phenotypes was significantly different among DAs and Non-DAs. Intestinal metaplasia was present in the surrounding mucosa of more than $90 \%$ of both DAs and Non-DAs. Surrounding mucosa of I/In-type was also frequently observed in DAs, as was that of I/Com-type in Non-DAs. The expression of p53 in DAs $(8 \%)$ was higher than that in Non-DAs (5\%). p53-positive adenomas were present only among the I/Com-type and I/Intype adenomas, while G-type and $U$-type adenomas showed no 553 positivity.

Conclusions. Both DAs and their surrounding mucosa appear to have an unstable status, because they possess a hybrid phenotype of I/In-type more commonly than Non-DAs. Such instability could be considered a risk factor for neoplastic potential and, therefore, DAs should be followed carefully.
\end{abstract}

Offprint requests to: M. Tsuneyoshi

Received: February 17, 2003 / Accepted: June 10, 2003
Key words Depressed adenoma - Gastric adenoma $\cdot$ Phenotypic expression · Gastric phenotype $\cdot$ Intestinal phenotype

\section{Introduction}

Most gastric carcinomas in an early phase show macroscopically depressed lesions [1]. A benign counterpart of depressed neoplasia of the stomach, which has hitherto been called depressed adenoma [2,3] (DA), consists of a glandular epithelium with varying degrees of cellular atypia and with papillary and/or tubular structures, similar to the features in Non-DAs [4]. Although controversy exists over whether or not DA is really adenoma [5], we adopted the term "adenoma" rather than "dysplasia," based on the description of the entity in the WHO publication [4]. Gastric DAs, which comprise approximately $10 \%$ of all gastric adenomas [2,3,6], have been reported to be either more aggressive or to have a higher malignant potential than ordinarily encountered Non-DAs [2,6,7].

Gastric carcinomas have been classified into two histological subtypes, "intestinal type" and "diffuse type" by Lauren [4]. The "diffuse type" is mainly characterized by poorly cohesive cells with diffuse infiltration of the gastric wall. On the other hand, the "intestinal type" is characterized by a predominance of glandular epithelium, often having intestinal-type cells, and it is almost identical to differentiated adenocarcinoma, while it is also associated with intestinal metaplasia in the vicinity of the tumor [4]. As regards genetic instability, intestinal metaplasia and gastric adenoma could share the genetic changes found in differentiated gastric carcinoma [8].

Recently, the cloning and sequencing of epithelial mucin genes has resulted in mucin histochemistry now being recognized as an accurate diagnostic modality for exploring the biological mechanisms underlying disor- 
ders of growth and differentiation $[9,10]$. So-called "second generation" monoclonal antibodies raised against the mucin core protein provide a useful guide to lineage differentiation of the gastrointestinal tract. MUC2 detects a gel-forming mucin specific to the goblet cell population. HGM, which is identical to MUC5AC, is another gel-forming mucin which is normally limited to the superficial and foveolar epithelium of gastric mucosa and mucous neck cells. In addition, CD10, which is identical to common acute lymphoblastic leukemia antigen (CALLA), is also considered to be useful for detecting the brush border in the small intestine. Expressions of these three different antibodies could be useful for assessing the intestinalization of tissues and evaluating their phenotypes more easily and objectively [11].

To elucidate the unique characteristics of gastric DAs, we examined the phenotypic expression of the neoplastic tissue and the surrounding mucosa, and compared our findings with those in Non-DAs.

\section{Materials and methods}

\section{Tissues studied}

We studied 50 lesions, diagnosed as DA, from 50 patients. These cases were selected from our files of gastric specimens which had been either surgically or endoscopically resected at Kyushu University Hospital or its affiliated hospitals. Forty-two Non-DAs from 42 patients were selected for a comparative study. Atypical changes in DAs and Non-DAs which consisted of cytological abnormalities, as well as changes in the architectural arrangement of glands, were determined by the authors (M.O. and T.Y.) to be of low grade according to the WHO classification (Fig. 1A,B,F,G). Therefore, all the adenomas were classified as noninvasive low-grade neoplasia (adenoma) in category 3 based on the Vienna classification [12]. Each representative paraffin block selected was cut into $4-\mu \mathrm{m}$-thick sections and was thus available for the immunohistochemical study.

Surrounding mucosa was defined as nonneoplastic mucosa within $5 \mathrm{~mm}$ from the tumor cell margins.

\section{Immunohistochemical staining}

To evaluate the phenotypic expression of the adenoma and the surrounding mucosa, the appearance of the brush border, goblet cells, and gastric foveolar epithelium was investigated by means of immunohistochemical staining, using monoclonal antibodies against HGM (1:50, 45M1; Novocastra, New Castle, UK) (Fig. 1C,H), MUC2 (1:200; Ccp58; Novocastra) (Fig. 1D,I), and CD10 (1:200; 56C6, Novocastra) (Fig. 1E,J). In addition, the immunohistochemical detection of p53 (1:100;
PAb1801; Oncogene, Darmstadt, Germany) was also carried out. The staining details have been described elsewhere [11]. In brief, sections were deparaffinized in xylene, hydrated through a graded series of ethanol, and immersed in $3 \% \mathrm{H}_{2} \mathrm{O}_{2}$ in absolute $100 \%$ methanol for $30 \mathrm{~min}$ to inhibit endogenous peroxidase. To retrieve antigens masked by formalin fixation, the slides for CD10, MUC2, and p53 were boiled in $10 \mathrm{mM}$ of $\mathrm{pH}$ 6.0 citrate buffer for $30 \mathrm{~min}$. After being rinsed in phosphate-buffered saline (PBS) and incubated with normal rabbit serum for $10 \mathrm{~min}$, the sections were incubated in humidified chambers with the primary antibody overnight at $4{ }^{\circ} \mathrm{C}$, followed by three washes with PBS. The streptavidin-biotin method, with Histofine SABPO (mouse) Kits (Nichirei, Tokyo, Japan) was used. The sections were then incubated with biotinylated rabbit anti-mouse immunoglobulin for $20 \mathrm{~min}$, followed by three washes with PBS. The slides were treated with peroxidase-conjugated streptavidin for $20 \mathrm{~min}$. After being washed in PBS, the slides were developed by immersion in $0.01 \% \mathrm{H}_{2} \mathrm{O}_{2}$ and $0.05 \% 3.3^{\prime}$-diaminobenzidine tetrahydrochloride (DAB) for $3 \mathrm{~min}$. Light counterstaining with Mayer's hematoxylin (CD10, MUC2, p53) or methyl green (HGM) was performed.

The results of staining for CD10, HGM, and MUC2 were categorized into two groups: positive expression and negative expression. The results of immunostaining were considered to be positive if more than $10 \%$ of the adenoma cells in the neoplastic areas or more than $10 \%$ of the tubules overall in the surrounding mucosa were stained. When $10 \%$ or fewer of the adenoma cells were stained, the results were considered to be negative. Regarding p53 expression, when more than $5 \%$ of the adenoma cells in the neoplastic areas were stained, it was evaluated as positive.

\section{Classification of phenotypic expression of adenoma and surrounding mucosa}

The classification of phenotypes of the adenoma and surrounding mucosa was based on the types of intestinal metaplasia (complete-type, incomplete-type, nonmetaplastic gastric mucosa). The phenotypes were classified into four categories according to the combination of the expression of the antibodies CD10 (brush border), MUC2 (goblet cell), and HGM (gastric foveolar epithelium), as described previously by our colleagues [11]. In brief, complete intestinal type (I/ Com-type) demonstrated positive expression for CD10 and was negative for HGM, regardless of MUC2 expression. Incomplete intestinal type (I/In-type) showed positive expression for both CD10 and HGM, or else negative expression for these two antibodies and positive expression only for MUC2. Gastric type (G-type) showed positive expression for HGM but negative ex- 

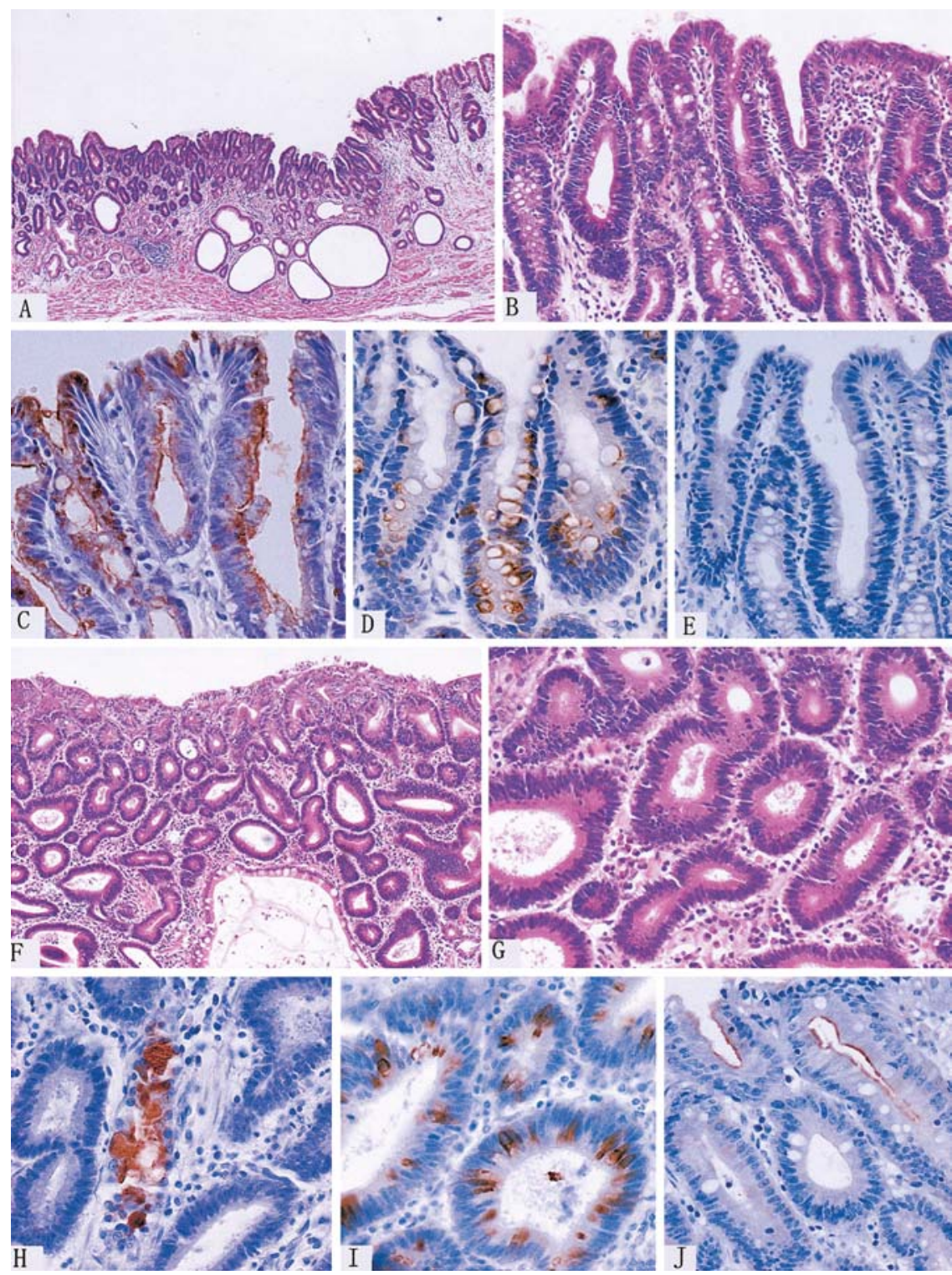

Fig. 1A-J. Depressed adenoma (DA) (A-E) and Non-DA (F-J). Adenomatous tubules with low-grade atypia were associated with several dilated pyloric glands underneath $(\mathbf{A}, \mathbf{F})$. A higher power view shows abundant relatively uniform glands (B, G). HGM staining is seen in the cytoplasm in most of the adenoma epithelium (C), but complete intestinal type (I/Comtype) Non-DA was negative for HGM staining, except for the nonneoplastic gland (H). MUC2 was positively expressed in goblet-like cells in neoplastic tissue $(\mathbf{D}, \mathbf{I})$. CD10 was positively expressed along the luminal surface of I/ Com-type Non-DA (J), while it was negative in incomplete intestinal type (I/In type) DA (E). A H\&E, $\times 20$; B H\&E, $\times 100$; C-E $\times 200 ;$ F H\&E, $\times 40$; G H\&E, $\times 100 ; \mathbf{H}-\mathbf{J} \times 200$ pression for CD10 and MUC2. Unclassified type (Utype) was negative for all of CD10, MUC2, and HGM. Table 1 summarizes these classifications.

\section{Statistical analysis}

Statistical analysis was conducted using Student's $t$-test and the $\chi^{2}$ test with Yates' correction. A $P$ value of less than 0.05 was considered to be statistically significant.

\section{Results}

\section{Clinicopathological features}

The clinicopathological features of the DAs are summarized in Table 2, together with those of the conventional
Table 1. Classification of phenotypes

\begin{tabular}{llcl}
\hline & & HGM-positive & HGM-negative \\
\hline \multirow{2}{*}{ CD10+ } & MUC2 + & I/In-type & I/Com-type \\
& MUC2- & I/In-type & I/Com-type \\
CD10- & MUC2+ & I/In-type & I/In-tpe \\
& MUC2- & G-type & U-type \\
\hline
\end{tabular}

Phenotypes were classified into four categories according to the combination of expression of CD10, MUC2, and HGM: I/Com-type, complete intestinal type; I/In-type, incomplete intestinal type; G-type, gastric type; U-type, unclassified type

Non-DAs. In this study, neither the DA nor the NonDA patients had multiple lesions. Both the DA and Non-DA patients were predominantly male, and in their late seventh decade. The mean size of the DAs was 
Table 2. Clinicopathologic differences between depressed adenoma and nondepressed adenoma

\begin{tabular}{lccc}
\hline & Depressed adenoma & Nondepressed adenoma & $P$ value \\
\hline Patients & & & \\
$\quad$ Number & 50 & 42 & \\
Age (years) range & $49-84$ & $48-79$ & NS \\
$\quad$ Mean \pm SD & $69.2 \pm 8.2$ & $68.1 \pm 6.8$ & NS \\
$\quad$ Sex (M:F) & $37: 13$ & $29: 13$ & \\
Lesions & $4-50 \mathrm{~mm}$ & $6-45 \mathrm{~mm}$ & NS \\
$\quad$ Size (diameter) & $14.0 \pm 10.1$ & $18.1 \pm 9.3$ & NS \\
Location \pm SD) & $1: 21: 28$ & $1: 25: 16$ & \\
$\quad$ Upper:middle:lower & & &
\end{tabular}

NS, not significant

Table 3. Phenotypes of adenomas

\begin{tabular}{lcc}
\hline & $\begin{array}{c}\text { Depressed } \\
\text { adenoma } \\
(n=50)\end{array}$ & $\begin{array}{c}\text { Nondepressed } \\
\text { adenoma } \\
(n=42)\end{array}$ \\
\hline Complete intestinal type & $19(38 \%)$ & $22(52 \%)$ \\
Incomplete intestinal type & $30(60 \%)$ & $15(36 \%)$ \\
Gastric type & $1(2 \%)$ & $1(2 \%)$ \\
Unclassified type & 0 & $4(10 \%)$
\end{tabular}

Depressed vs nondepressed adenoma, $P<0.05$

smaller than that of the Non-DAs, but the difference was not statistically significant. Concerning the location within the stomach, most DAs were located in the lower third, whereas the Non-DAs were present predominantly in the middle third.

\section{Phenotypic expression in adenomas and surrounding mucosa}

CD 10 staining was seen along the brush border of the luminal surface of the epithelium. Although CD 10 can also be expressed in the apical portion of the cytoplasm of normal gastric mucosa, only the expression of CD10 on the brush border was studied. MUC2 staining was seen in the cytoplasm around the nuclei of goblet cells. HGM staining was seen in the cytoplasm of the aforementioned cells.

Table 3 shows the phenotypic expression of the DAs and Non-DAs. I/In-type adenomas were frequently observed among DAs, whereas I/Com-type adenomas were frequently seen in Non-DAs. The incidence of phenotypes was significantly different among DAs and Non-DAs.

The phenotypic expression of the mucosa surrounding both DAs and Non-DAs, in terms of intestinalization, is summarized in Table 4. Complete or incomplete intestinal was observed in metaplasia the
Table 4. Phenotypes of the surrounding mucosa

\begin{tabular}{lcc}
\hline & $\begin{array}{c}\text { Depressed } \\
\text { adenoma } \\
(n=50)\end{array}$ & $\begin{array}{c}\text { Nondepressed } \\
\text { adenoma } \\
(n=42)\end{array}$ \\
\hline Complete intestinal type & $15(30 \%)$ & $21(50 \%)$ \\
Incomplete intestinal type & $32(64 \%)$ & $19(45 \%)$ \\
Gastric type & $3(6 \%)$ & $2(5 \%)$ \\
Unclassified type & 0 & 0
\end{tabular}

Depressed vs nondepressed adenoma, $P>0.05$

surrounding mucosa of more than $90 \%$ of both DAs and Non-DAs. There was a high incidence of I/In-type in the surrounding mucosa of DAs, as well as a high incidence of I/Com-type in the surrounding mucosa of Non-DAs.

As for a detailed correlation between the phenotype of adenoma and that of its background mucosa, I/Intype mucosa was overwhelmingly associated with I/Intype adenoma in DAs (24/32; 75\%). I/In-type mucosa in Non-DAs was associated with I/In-type adenomas (9/ $19 ; 47 \%)$, I/Com-type ones $(8 / 19 ; 42 \%)$, and others. I/ Com-type mucosa was largely correlated with I/Comtype adenoma both in DAs $(10 / 15 ; 67 \%)$ and Non-DAs $(14 / 21 ; 67 \%)$.

\section{Correlation between phenotype and 553 expression}

p53-positive cases were detected in I/Com-type (1/19; $5 \%)$ and I/In-type $(3 / 30 ; 10 \%)$ DAs, and in I/Com-type $(1 / 22 ; 5 \%)$ and I/In-type $(1 / 15 ; 7 \%)$ Non-DAs as well. The incidence of $\mathrm{p} 53$ expression in DAs $(4 / 50 ; 8 \%)$ was higher than that in Non-DAs $(2 / 42 ; 5 \%)$, but the difference was not statistically significant. The incidence of p53-positive cases was not significantly different among the four phenotypes in either DAs or Non-DAs. However, p53 expression was present only in I/Comtype and I/In-type adenomas, while adenomas lacking 
intestinalization (G-type and U-type) showed no immunohistochemical positivity for $\mathrm{p} 53$ expression.

\section{Discussion}

The histological, histochemical, and various immunohistochemical profiles of DAs have been previously reported by several authors $[2,3,6,7]$. In those reports, the incidence of carcinoma in adenoma was demonstrated to be higher in DAs than in conventional Non-DAs, and thus DAs are considered to be a variant of gastric adenoma carrying a somewhat higher malignant potential $[2,6,7]$. Gastric adenomas have been reported to be related to intestinal metaplasia of the gastric mucosa $[8,13]$. The majority of gastric adenomas have epithelia showing intestinal features [4]. However, conventional histochemical staining may lead to some difficulty in interpreting the phenotypes of tissues [14]. Recently developed antibodies for mucin core proteins have been shown to be a useful guide to altered lineage differentiation of gastrointestinal epithelium and epithelial neoplasia $[9,10]$. With CD10, we could objectively evaluate the presence of a brush border. In the present study, the incidence of I/In-type adenoma was significantly higher in DAs than in Non-DAs, thus indicating DAs to be unique neoplasms which could be different from NonDAs. A frequent occurrence of the I/In-phenotype in neoplastic tissue as well as in the surrounding mucosa implies that DAs carry an unstable form of intestinalization [10], which may be closely correlated to its genetic instability [9]. Our colleagues have also reported a similar outcome in multiple gastric cancers compared with solitary cancers [11]. We found here that, in Non-DAs the I/Com-type was the predominant phenotype both in neoplastic tissue and in the surrounding mucosa, and this type is thus considered to be relatively stable. However, the I/In-type in surrounding mucosa of Non-DAs is considered to be unstable because it is associated with adenomas of various phenotypes.

Intestinal metaplasia is a common phenomenon in the human stomach, and its prevalence increases with age, while it is also associated with chronic atrophic gastritis, peptic ulcer [15], and Helicobacter pylori infection. In this study, intestinal metaplasia was overwhelmingly present in the surrounding mucosa irrespective of any morphological differences in the gastric adenomas. Several recent studies have disclosed that intestinal metaplasia may be paraneoplastic rather than preneoplastic, and an intestinal phenotype in differentiated cancers might reflect the phenotypic status of their surrounding mucosa $[16,17]$. In this study as well, the speculation that tumor tissues reflect the phenotypes of the surrounding mucosa cannot be totally ignored. However, the significant difference between the pheno- typic expression in DAs and Non-DAs would appear to be based on something in addition to the different surrounding mucosa, because changes in various genes determining cell structures and functions have been involved in intestinal metaplasia $[8,18]$. The genetic alterations involved in the development of DAs and Non-DAs might, thus, be partly different. In addition, the intestinal phenotype was suggested to not only be independent of intestinal metaplasia in the surrounding mucosa but it was also suggested to show a kind of time-dependent quality, at least in gastric signet-ring cell carcinoma [19]. The high incidence of In/Com-type in Non-DAs might reflect its longstanding presence in the stomach. The incidence of both DAs and NonDAs having gastric-type phenotype was very low in our study. However, it is possible that the incidence of his phenotype could have been higher if markers of pyloricgland mucin (CPS III [19], HIK1083 [20] or MUC6 $[9,10])$ had been used.

No significant correlation between p53 expression and morphological aspects was observed. Although we did not confirm p53 gene alteration or p21/waf1 [21] expression status in neoplastic tissue, our finding of lack of correlation, above, might have been affected by the presence of various genetic pathways in the tumorigenesis of gastric adenomas besides $p 53$ gene alteration $[8,13]$. The frequent occurrence of microsatellite instability has been indicated previously in gastric foveolar-type adenomas [22]. Gastric-type adenomas and unclassified-type ones, non-intestinalized phenotypes, had no p53-positive cases in DAs or Non-DAs, although the number of cases we examined in this study was relatively small. This finding is similar to that in a previous study, in which $p 53$ gene alteration was not considered to be an early event in the gastric carcinogenetic sequence with a gastric phenotype [23].

In conclusion, most DAs and their surrounding mucosa demonstrated an I/In-type phenotype, which is a hybrid phenotype of the stomach and small intestine. As a result, DAs are more unstable than Non-DAs, which are composed predominantly of I/Com-type tissues. Such instability is considered to be a risk factor for neoplastic potential, and, therefore, DAs should be followed carefully.

Acknowledgments The authors thank Brian Quinn for reviewing the English language in the manuscript.

\section{References}

1. Eckardt VF, Gießler W, Kanzler G, Remmele W, Bernhard G. Clinical and morphological characteristics of early gastric cancer. Gastroenterology 1990;98:708-14.

2. Nakamura K, Sakaguchi H, Enjoji M. Depressed adenoma of the stomach. Cancer 1988;62:2197-202. 
3. Ito H, Yasui W, Yoshida K, Nakayama H, Tahara E. Depressed tubular adenoma of the stomach: pathological and immunohistochemical features. Histopathology 1990;17:419-26.

4. Watanabe H, Jass JR, Sobin LH. WHO histological typing of oesophageal and gastric tumours. (2nd Ed). Berlin Heidelberg New York Tokyo: Springer-Verlag; 1990. pp 19-39.

5. Goldstein NS, Lewin KJ. Gastric epithelial dysplasia and adenoma: historical review and histological criteria for grading. Hum Pathol 1997;28:127-33.

6. Tsujimani S, Furusawa M, Hayashi I. Morphological factors aid in therapeutic decisions concerning gastric adenomas. Hepatogastroenterology 1992;39:56-8.

7. Xuan ZX, Ambe K, Enjoji M. Depressed adenoma of the stomach, revisited; histologic, histochemical, and immunohistochemical profiles. Cancer 1991;67:2382-9.

8. Tahara E. Molecular biology of gastric cancer. World J Surg 1995; 19:484-90.

9. Jass JR. Mucin core proteins as differentiation markers in the gastrointestinal tract. Histopathology 2000;37:561-4.

10. Jass JR, Walsh MD. Altered mucin expression in the gastrointestinal tract: a review. J Cell Mol Med 2001;5:327-51.

11. Kabashima A, Yao T, Sugimachi K, Tsuneyoshi M. Gastric or intestinal phenotypic expression in the carcinomas and background mucosa of multiple early gastric carcinomas. Histopathology 2000;37:513-22.

12. Schlemper RJ, Riddell RH, Kato Y, Borchard F, Cooper HS, Dawsey SM, et al. The Vienna classification of gastrointestinal epithelial neoplasia. Gut 2000;47:251-5.

13. Tamura G. Molecular pathogenesis of adenoma and differentiated adenocarcinoma of the stomach. Pathol Int 1996;46:83441.

14. Yao T, Kabashima A, Kouzuki T, Oya M, Tsuneyoshi M. The phenotypes of the gastric carcinoma; evaluation by a new immunohistochemical method. I to Cho (Stomach and Intestine) 1999; $34: 477-85$.
15. Filipe MI, Munoz N, Matko I, Kato I, Pompe-Kirn V, Jutersek A, et al. Intestinal metaplasia types and the risk of gastric cancer: a cohort study in Slovenia. Int J Cancer 1994;57:324-9.

16. Tabata H, Fuchigami T, Yao T, Kobayashi H, Sakai Y, Iizuka Y, et al. Clinicopathological study of gastric adenoma in relation to two different gastric gland areas. I to Cho (Stomach and Intestine) 1997:32:1591-7.

17. Matsukuma A, Mori M, Enjoji M. Sulphomucin-secreting intestinal metaplasia in the human gastric mucosa; an association with intestinal-type gastric carcinoma. Cancer 1990;66:689-94.

18. Silberg DG, Furth EE, Taylor JK, Schuck T, Chiou T, Traber PG. CDX1 protein expression in normal, metaplastic, and neoplastic human alimentary tract epithelium. Gastroenterology 1997;113: 478-86.

19. Bamba M, Sugihara H, Kushima R, Okada K, Tsukashita S, Horinouchi M, et al. Time-dependent expression of intestinal phenotypein signet ring cell carcinomas of the human stomach. Virchows Arch 2001;438:49-56.

20. Ota H, Hayama M, Nakayama J, Hidaka H, Honda T, Ishii K, et al. Cell lineage specificity of newly raised monoclonal antibodies against gastric mucins in normal, metaplastic, and neoplastic human tissues and their application to pathology diagnosis. Am J Clin Pathol 2001;115:69-79.

21. Oya M, Yao T, Tsuneyoshi M. Expressions of cell-cycle regulatory gene products in conventional gastric adenomas: possible immunohistochemical markers of malignant transformation. Hum Pathol 2000;31:279-87.

22. Endoh Y, Sakata K, Tamura G, Ohmura K, Ajioka Y, Watanabe $\mathrm{H}$, et al. Cellular phenotypes of differentiated-type adenocarcinomas and precancerous lesions of the stomach are dependent on the genetic pathways. J Pathol 2000;191:257-63.

23. Kushima R, Muller W, Stolte M, Borchard F. Differential p53 protein expression in stomach adenomas of gastric and intestinal phenotypes: possible sequences of p53 alteration in stomach carcinogenesis. Virchows Arch 1996;428:223-7. 